\title{
Polarization-Sensitive CARS of the Amide I Band of Pure and Liganded Chymotrypsin
}

\author{
A. Yu. Chikishev* and N. I. Koroteev \\ International Laser Center and Physics Department Moscow State University, 119899 Moscow, Russia \\ C. Otto* and J. Greve \\ Applied Optics Group, University of Twente, PO Box 217, NL-7500 AE Enschede, Netherlands
}

\begin{abstract}
Polarization-sensitive coherent anti-Stokes Raman scattering (PSCARS) is used to investigate the secondary structure of the protein chymotrypsin, both free and bound to antranilic acid. Advantage is taken of the extreme sensitivity of the PSCARS spectra to the orientation of the analyser. Clear changes are observed in the protein spectra as a result of binding to antranilic acid. It is concluded that PSCARS can be fruitfully applied to detect changes in the bonds of the peptide backbone of enzymatic proteins as a result of substrate binding.
\end{abstract}

\section{INTRODUCTION}

It was demonstrated earlier that polarization-sensitive coherent anti-Stokes Raman scattering (PSCARS) may provide direct spectroscopic information on the structure of the broad conformationally sensitive amide I band in the vibrational spectra of proteins. ${ }^{1}$ The choice of appropriate polarization conditions allowed us to detect three bands constituting the broad amide I band. We related the subbands observed to the elements of the secondary structure ( $\alpha$-helix, $\beta$-sheet, random coil) for several proteins. We demonstrated that there was a good correlation between the PSCARS and alternative ( $x$-ray diffraction, $C D$ and spontaneous Raman) data on the secondary structure content in various proteins under study. In the present work we compare the PSCARS spectra of the amide I band of the free enzyme (chymotrypsin) in solution with that of the liganded enzyme in solution. The changes observed are assigned to the conformational change of the enzyme molecule upon ligand binding.

\section{EXPERIMENTAL}

Coherent anti-Stokes Raman scattering (CARS) is a four-wave mixing process. CARS is based on the coherent excitation of molecular vibrations in a sample by two waves $\left(\omega_{1}, \omega_{2}\right)$ probed by the third wave $\left(\omega_{3}\right)$. This interaction results in the appearance of coherently scattered radiation in the anti-Stokes part of the spectrum $\left(\omega_{\mathrm{a}}\right)$. The scattered signal undergoes resonance enhancement when the frequency difference of the first two

\footnotetext{
* Authors to whom correspondence should be addressed.
}

waves equals the frequency of a molecular vibration $\left(\omega_{1}\right.$ $\left.-\omega_{2}=\omega_{\mathrm{vib}}\right)$. In our experiments we used the degenerate scheme where $\omega_{3}=\omega_{1}$ and the frequency of the scattered signal is $\omega_{a}=\omega_{1}+\left(\omega_{1}-\omega_{2}\right)=2 \omega_{1}-\omega_{2}$. The experimental set-up has been described elsewhere. ${ }^{1,2}$ The pump beams $\left(\omega_{1}, \omega_{2}\right)$ were provided by a Quanta Ray DCR 2 pulsed Nd: YAG laser (second harmonic $\omega_{1}=532 \mathrm{~nm}, 10 \mathrm{~Hz}$ repetition rate) and a PDL2 dye laser which contained kiton red and operated within the wavelength range $578-606 \mathrm{~nm}$, thus providing CARS frequency detunings corresponding to a Stokes shift of $1500-2200 \mathrm{~cm}^{-1}$. The energies at the sample in the pumping beams were $400 \mu \mathrm{J}$ pulse $^{-1}$. Application of the polarization-sensitive variant of CARS is dictated by the presence of a strong coherent non-resonant background in the signal. The polarizations of the beams were set by high-quality GlanTaylor prisms (extinction ratio $10^{-7}$ at crossed polarizations). The angle between polarizations $P 1$ and P2 $(F)$ was chosen within the range $60^{\circ}-70^{\circ}$. The CARS signal was analysed by a Glan-Taylor polarizing prism. This polarization analyser was set at an angle $Y$ relative to the polarization $\mathrm{P} 1$. The values of $Y$ were chosen close to that providing polarization suppression of the non-resonant background. Additional experimental and theoretical considerations of the principles of CARS and its application to the study of biological molecules can be found in Refs 1-4. It is stressed here that CARS experiments carried out on solutions of biological molecules require a very high quality of the degree of polarization of the pumping beams. This is extremely critical for high-quality non-resonant background suppression.

Chymotrypsin (CT) was purchased from Sigma. The liganded enzyme [chymotrypsin complex with antranilic acid (CT-AA)] was prepared according to Ref. 5. Both the pure and the liganded enzyme were dissolved in heavy water to obtain a protein concentration of $100-150 \mathrm{mg} \mathrm{ml}^{-1}$. Heavy water was used to avoid the overlap of vibrations of normal water and the amide I band in the $1600-1700 \mathrm{~cm}^{-1}$ region. According to Blout et al., ${ }^{6} 85 \%-90 \%$ deuterium exchange with amide 
protons takes place within $3 \mathrm{~h}$. The PSCARS spectra were recorded not earlier than $3 \mathrm{~h}$ after sample preparation. Until that time the solutions were kept in a refrigerator under sterile conditions at $4{ }^{\circ} \mathrm{C}$. The front and back windows of the sample cells $(1.0-2.0 \mathrm{~mm}$ thick) were made of microscope cover glass slides, which were antireflection coated at the glass-air interface to minimize multiple-interference effects.

\section{RESULTS}

PSCARS spectra of free and liganded chymotrypsin heavy water solutions were measured at various settings of the polarization analyser, $Y$. We also carried out measurements of PSCARS spectra of antranilic acid heavy water solutions. No vibrational bands were observed within the wavenumber range of interest. Hence all the spectral features observed in CT-AA heavy water solution PSCARS spectra belong to the protein. In Fig. 1 we demonstrate the reproducibility of the PSCARS spectra of chymotrypsin heavy water solutions. The spectra were recorded several days apart, during which time the set-up was readjusted several times. Although PSCARS spectra are very sensitive to the beam configuration, we observe that the reproducibility of the spectra is high and we can trust spectral differences exceeding approximately $10 \%$ as reliable indications of molecular changes. In Fig. 2 the PSCARS spectra of CT heavy water solutions are presented. The spectra are measured under different polarization conditions. The large changes in the band shapes result from the interference character of the CARS signal. Even minor changes in polarization conditions lead to dramatic changes in the spectral features. The interpretation of the spectral changes is only possible after fitting all the spectra obtained. The fitting procedure developed earlier $^{1,2}$ allows us to carry out the simultaneous

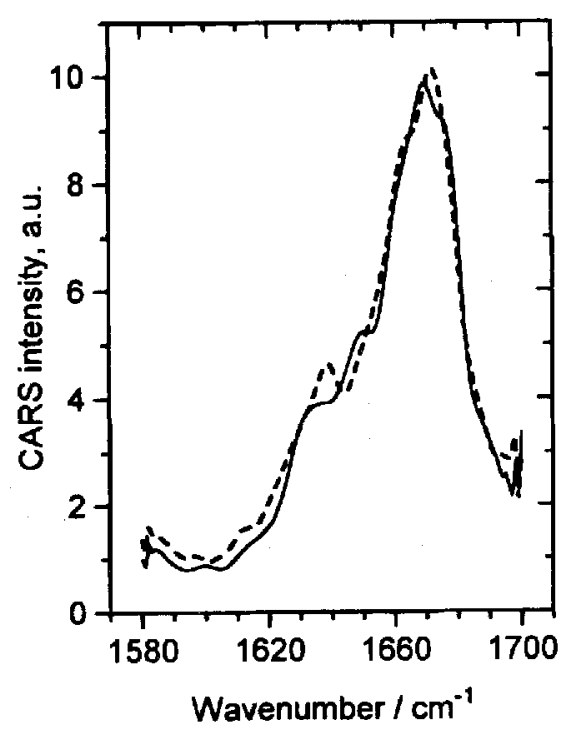

Figure 1. PSCARS spectra of chymotrypsin dissolved in deuterated water solution measured on different days under the same polarization conditions $\left(F=60^{\circ}, Y=62.63^{\circ}\right)$ to test data reproducibility: full-curve, measurement 1; broken curve, measurement 2.

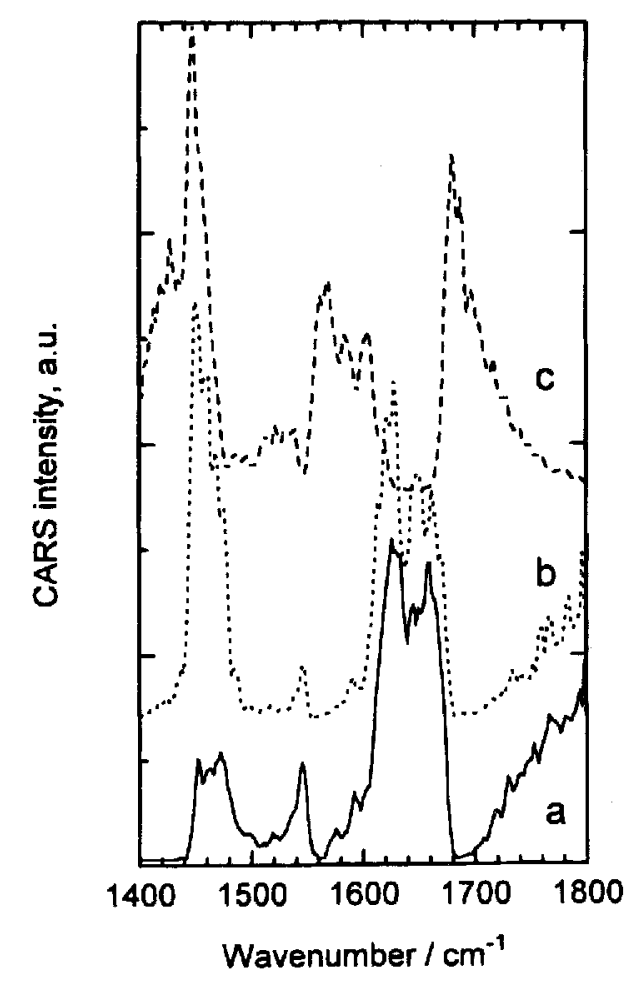

Figure 2. PSCARS spectra of chymotrypsin dissolved in deuterated water solution measured under different polarization conditions: a, $F=69.69^{\circ}, Y=50.31^{\circ} ; b, F=70.24^{\circ}, Y=49.76^{\circ}$; c, $F=71.88^{\circ}, Y=48.12^{\circ}$

fit of several PSCARS spectra, measured at different $Y$, with one set of vibrational band parameters (amplitudes, wavenumbers), bandwidths, depolarization ratios). It was demonstrated ${ }^{2}$ that multispectra fitting increases significantly the accuracy of the procedure. In Fig. 3 we compare the PSCARS spectra of CT (full curve) and CT-AA (broken curve) heavy water solutions at two different positions of the analyser. There are clear differences between the spectra of the native and the liganded enzyme which significantly exceed the experimental error (see Fig. 1). The fitting procedure for two groups of spectra was applied in the following way. At first a fit was carried out of CT heavy water solution PSCARS spectra with the set of vibrational band parameters determined earlier in Ref. 1. The spectra could be nicely fitted with approximately the same band
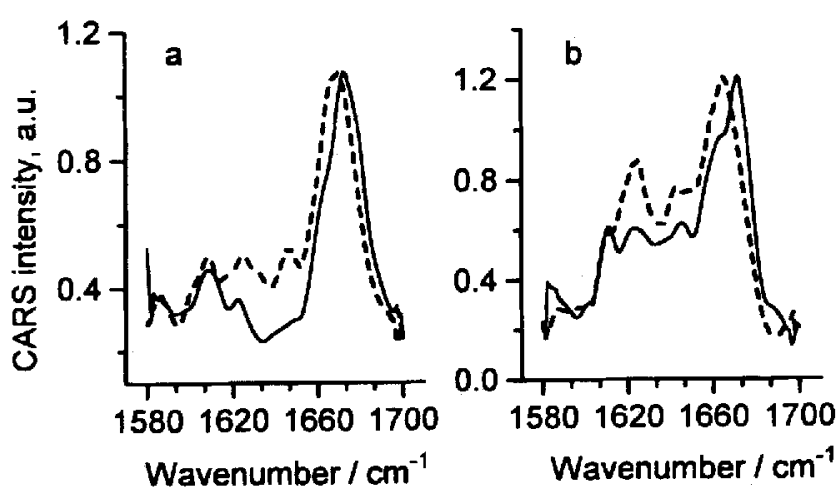

Figure 3. PSCARS spectra of chymotrypsin (full curve) and chymotrypsin-antranilic acid (broken curve) dissolved in deuterated water solution: $a, F=60^{\circ}, Y=62.88^{\circ} ; b, F=60^{\circ}, Y=62.75^{\circ}$. 


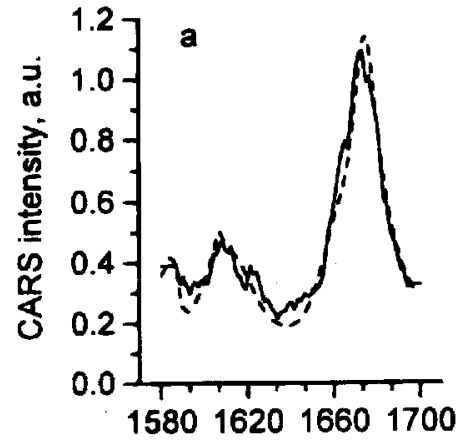

Wavenumber $/ \mathrm{cm}^{-1}$

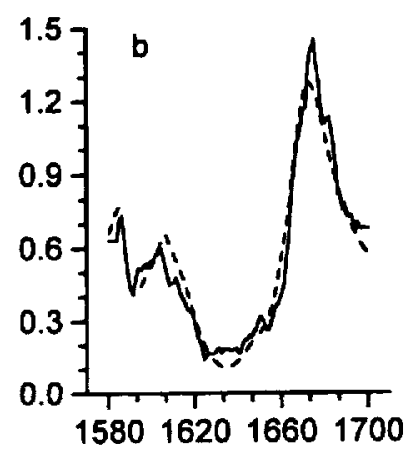

Wavenumber $/ \mathrm{cm}^{-1}$

Figure 4. Comparison of fitting results with experimental PSCARS spectra: a, fit (broken curve) of the PSCARS spectrum of chymotrypsin in deuterated water solution, with $F=60^{\circ}, Y=62.88^{\circ} ; b$, fit of the PSCARS spectrum of chymotrypsin-antranilic acid in deuterated water solution, with $F=60^{\circ}, Y=63.13^{\circ}$.

parameters as determined in Ref. 1 . Thus accurate band parameters can be obtained for the bands constituting the amide I band as well as for the adjacent bands assigned to the vibrations of aromatic amino acids and $\mathrm{CH}_{2} \mathrm{CH}_{3}$ groups. The result of the fit for one of the spectra is demonstrated in Fig. 4a. After that we started the fitting procedure for CT-AA heavy water solution PSCARS spectra with the set of parameters obtained for the native enzyme. Only the bands constituting the amide I band changed their parameters during the fit, whereas the parameters for the neighbouring bands

\begin{tabular}{|c|c|c|c|}
\hline No. & Wavenumber $/ \mathrm{cm}^{-1}$ & Bandwidth $/ \mathrm{cm}^{-1}$ & Amplitude/8.u. \\
\hline \multicolumn{4}{|c|}{ CT heavy water solution } \\
\hline 1 & 1639.2 & 10.0 & 0.98 \\
\hline 2 & 1661.8 & 13.3 & 16.95 \\
\hline 3 & 1673.1 & 7.5 & 19.77 \\
\hline \multicolumn{4}{|c|}{ CT-AA heavy water solution } \\
\hline 1 & 1646.7 & 10.1 & 9.79 \\
\hline 2 & 1664.3 & 13.8 & 21.13 \\
\hline 3 & 1607.2 & 9.9 & 17.79 \\
\hline
\end{tabular}

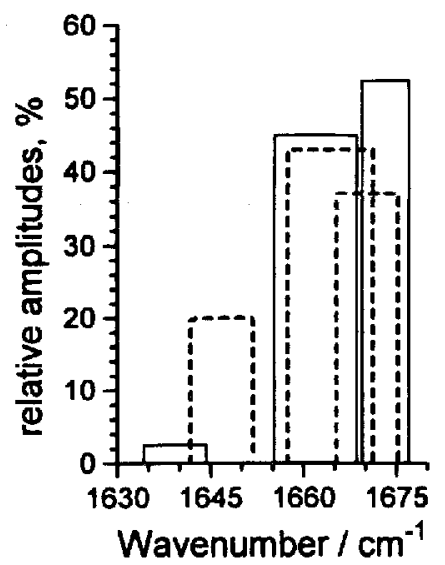

Figure 5. Results from the fit of the vibrational bands in PSCARS spectra of chymotrypsin (full line) and chymotrypsin-antranilic acid (broken line) dissolved in deuterated water solution (see text for details). remained within the error margin. The result of the fit for CT-AA is presented in Fig. 4b. In Table 1 we present the parameters obtained after the fitting procedure. All the bands could only be fitted as polarized $\left(0<R<R_{\text {non-resonant }}=\frac{1}{3}\right)$. The exact values of $R$ for the bands of interest could not be determined because of the very narrow range of $Y$ variation. However, as was demonstrated in Ref. 1, the estimation of the content of the secondary structure elements can be reliably performed. In Fig. 5 we present a histogram with the parameters of the vibrational bands obtained after the fitting procedure. The positions of the columns in the histogram and their widths correspond to the vibrational band positions and bandwidths. Each amplitude was normalized to the sum of the three amplitudes in the corresponding spectra. Thus the vertical axis gives information on the secondary structure element content, assuming that each band observed in the PSCARS spectra corresponds to some element of the secondary structure. Since the PSCARS spectral shapes differ a lot from that in spontaneous Raman, it may be more convenient to present the results of the fit in the form of spontaneous Raman spectra and/or their difference. As all the parameters of the vibrational bands are available after the fit, it is possible to calculate spontaneous Raman spectra. To stress the differences in the vibrational spectra of the samples under study, we present in

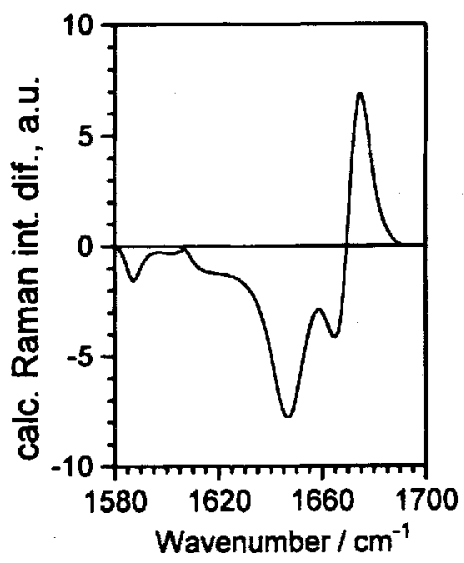

Figure 6. Difference spectrum of the calculated Raman spectra of chymotrypsin and chymotrypsin-antranilic acid heavy water solutions (see text for details). 
Fig. 6 the difference of the calculated spontaneous Raman spectra of pure and liganded enzyme heavy water solutions. The spontaneous Raman spectra are calculated from the fit of the PSCARS spectra.

\section{DISCUSSION}

It is well known that the enzyme molecule changes conformation upon ligand binding. ${ }^{7}$ It is quite clear that the conformational change takes place first of all at the active site. However, one may assume that not only the active site-a rather small part of the protein molecule - is involved in the conformational changes, but the protein molecule as a whole. In the present study we examined only one band sensitive to the secondary structure of the protein molecule or, more specifically, to the state of the peptide bonds in the macromolecule. As there are pronounced changes in the protein spectra in the amide I range upon ligand binding, it is natural to suggest that the conformational changes influence the whole molecule. The interpretation of the spectral changes observed and their correlation with the secondary structure seem to be rather difficult. In our earlier work we have shown that there is a good correlation of our PSCARS data on the secondary structure of various proteins with data obtained by alternative methods. Assuming that the three bands observed in the PSCARS spectra correspond to three main elements of the secondary structure, we arrive in our case at the conclusion that the ligand binding results in a significant decrease in the $\beta$-sheet content and increase in the $\alpha$-helix content. The content of the random coil remains practically unchanged. However, there is an alternative interpretation. The very notion of elements of the secondary structure is somewhat ideal and is based on analysis of the $x$-ray data: the values of torsion angles, $\mathrm{H}$ bond lengths and $\mathrm{C}_{\alpha}-\mathrm{C}_{\alpha}$ distances. $^{8}$ These characteristic parameters are usually grouped around some mean values, so the elements of the sec- ondary structure are formally nothing but these groups. Sometimes the dispersion of the parameters becomes high and the assignment at the level of the secondary structure becomes rather arbitrary. We propose an analogous (to that of the $x$-ray data) interpretation of our PSCARS data. What we observe in the experiments on vibrational spectroscopy is the state of the peptide bonds. It follows from our experimental data that these bonds form three groups and those inside a group possess similar Raman scattering tensors. From this point of view the spectral changes observed show that a rather large part of the peptide bonds in the protein molecule undergoes changes upon ligand binding. We may conclude that the comparison of PSCARS spectra of the amide I band of pure and liganded enzymes provides information on the conformational changes of the protein molecule involving changes in the intramolecular peptide bonds.

\section{CONCLUSION}

PSCARS spectra of the amide I band in chymotrypsin are very sensitive to the exact position of the analyzer. Binding of the protein to the substrate antranilic acid gives rise to large changes in the PSCARS spectra. A quantitative analysis is made of the spectra by a computer programme that simultaneously fits all obtained polarization-sensitive CARS spectra to a single set of parameters. It is concluded that the intramolecular peptide bonds in the protein have changed as can be observed in the amide I region.

\section{Acknowledgements}

The research was supported by NWO (Dutch Organisation for the Advancement of Science). The authors are indebted to Dr Inna Sakodynskaya for preparation of the samples and to Dr Gerald W. Lucassen and Dr Bulat N. Toleutaev for stimulating discussions and development of the software for fitting the PSCARS spectra.

\section{REFERENCES}

1. A. Yu. Chikishev, G. W. Lucassen, N. I. Koroteev, C. Otto and J. Greve, Biophys. J. 63, 976 (1992).

2. A. Voroshilov, G. W. Lucassen, C. Otto and J. Greve, J. Raman spectrosc. 26, 443 (1995).

3. S. A. Akhmanov and N. I. Koroteev, Methods of Nonlinear Optics in Light Scattering Spectroscopy. Nauka, Moscow (1991).

4. W. Kiefer, in Infrared and Raman Spectroscopy: Methods and Applications, edited by B. Schrader, p. 162 . VCH, Weinheim (1995).
5. L. Stryer, Science 162, 526 (1968); V. N. Dorovska-Taran, E. Veeger and A. J. W. F. Visser, Eur. J. Biochem. 218, 1013 (1993); 221,47 (1993).

6. E. R. Blout, C. DeLoae and A. Asadourian, J. Am. Chem. Soc 83, 1895 (1961).

7. A. Fersht, in Enzyme Structure and Mechanism. W. H. Freeman, San Francisco, CA (1977).

8. M. Levitt and J. Greer, J. Mol. Biol. 114, 181 (1977). 